\title{
Time to Teach: Addressing the Pressure on Faculty Time for Education
}

\author{
Adam M. Brenner ${ }^{1} \cdot$ Eugene V. Beresin $^{2}$. John H. Coverdale ${ }^{3}$ - Alan K. Louie ${ }^{4}$. \\ Richard Balon ${ }^{5}$ - Anthony P. S. Guerrero ${ }^{6}$. Laura Weiss Roberts ${ }^{4}$
}

Received: 27 October 2017 / Accepted: 1 November 2017/Published online: 13 November 2017

(C) Academic Psychiatry 2017

We are at a potentially opportune moment to address the longstanding issue of whether and how to protect time for faculty to teach, in part because of growing appreciation of the reality of faculty burnout and the ACGME mandate to monitor faculty wellness [1]. Burnout, which includes emotional exhaustion, depersonalization (experiencing people as objects), reduced empathy, and a diminished sense of personal accomplishment in one's work [2] is widespread among faculty [3]. Possible causes of burnout include the excessive size of patient panels and lack of time for individual patients, diminished reimbursements, greater bureaucratic demands, an overall decrease in what physicians actually find personally rewarding, and "an erosion of meaning in work" [4]. Shanafelt reported on a "growing body of research suggesting that enhancing meaning in work increases physician satisfaction and reduces burnout" [5]. Similarly, Pololi et al. found that the best predictors of faculty feeling energized and vital at work are feeling included/belonging, strong relationships, institutional support for their career, and alignment of values [6].

What is valued and meaningful will vary from physician to physician (and may include increased time with patients, interaction with colleagues, the ability to undertake research and

Adam M. Brenner

adam.brenner@utsouthwestern.edu

University of Texas Southwestern Medical Center, Dallas, TX, USA

2 Harvard Medical School, Boston, MA, USA

Baylor College of Medicine, Houston, TX, USA

4 Stanford University, Stanford, CA, USA

Wayne State University, Detroit, MI, USA

6 University of Hawai'i John A. Burns School of Medicine, Honolulu, HI, USA be involved meaningfully in policy and administration, etc.), but for academic physicians, it often involves time for education. Indeed, many faculty become academic psychiatrists precisely because they are dedicated to teaching. After all, the word doctor derives from the Latin docore, meaning to teach, and underscores the value attributed to transmitting knowledge to patients, students and physicians in training, and the wider society by joining the profession. At a time when the ACGME is focusing on the Clinical Learning Environment [7], it is worth noting that the least visible but perhaps most essential element of that environment is time [8]. Our goal for this editorial is to describe some of the factors, particularly financial pressures, that impose on teaching time and to place these in a historical perspective. We also wish to discuss some options for rectifying the problems that we identify.

\section{Financial Pressures Impeding Teaching}

Faculty commonly express the frustration that they do not have enough time to teach. This is not a new problem. It is rooted in the economic structure of academic medicine that became particularly critical following the managed care reconstruction of the healthcare system in the 1980s. In surveying the history of the past century of medical education, Barzansky wrote, "In general, there has been no clear picture of what each of the three medical school missions' - research, education, and patient care - cost or of what sources of revenue are used to support them" [9]. The individual faculty member has some vague idea that the three missions may cross-subsidize each other, but this is often done without transparency, and sometimes in a manner that leaves education viewed as a drain on the department. Although substantial funding, including Medicare payments and state support, is generated by teaching activities and earmarked for education, 
a lack of transparency at many institutions makes it impossible for educators to know where and how these education funds are applied. One of the authors recalls working in an academic department where education was explicitly called a "loss center" (as opposed to the department's "profit centers"), illustrating an often unquestioned assumption that education is not pulling its weight in terms of bringing in financial support when the opposite may be true. It is not surprising if faculty feel a tension and lack of clarity about how education is to be supported. Faculty may feel that even time that is allegedly available for teaching is not protected for this purpose.

The reason for this threatened status is very understandable in that teaching takes time and time is money. Unfortunately, academic medical centers are facing significant financial pressures. Recent stresses include reductions in Medicare and Medicaid reimbursements, insurer strategies to drive enrollees to lower cost community providers, primary care and psychiatric workforce shortages leading to increased utilization of high-cost emergency departments, and elimination of some sources of federal and state funding for clinical and research activities. These forces have led to the contemplation of shifts in medical education toward using dedicated instructors (such as a core clinical faculty), increased online instruction, simulation, virtual reality, and even gaming, and have led to an examination of shortening the training time [10] (p.993).

Certainly, academic physicians should take advantage of every possible opportunity to increase educational efficiency in close concert with the balance of evidence for doing so. There are potential efficiencies to be pursued, such as using "flipped classroom" experiences with self-study and guided, small group, team-based critical thinking or leveraging online resources (such as the National Neuroscience Curriculum Initiative) to supplement face-to-face clinical teaching. Pursuit of such efficiencies may allow schools to focus faculty teaching time where it adds the most value [11].

There is an inevitable limit to such efficiencies, however, and some of these attempts at modernizing medical education may actually increase faculty teaching time. For example, flipped classroom discussions and team-based learning require moderators to help small groups focus their thinking and require a considerable amount of preparatory time. Moreover, becoming a physician, or a psychiatrist, is too complex and nuanced a task to be learned solely through self-study. Most of us will easily remember the crucial experience of hearing an attending on the wards explain her thinking or demonstrate a clinical skill. As Kahn et al. argued, excellent teaching will remain crucial in an era of computer-assisted diagnosis and treatment planning and should focus on developing the ability to translate "machine-generated recommendations into personalized care attuned to the specific context of a patient" [11] (p. 1216).
In a similar vein, Skeff et al. responded to calls for more efficient teaching by cautioning that teaching is necessarily time consuming, as it includes planning, instructing, and reflecting [12]. They argued that for teaching to be optimal, it needs to move beyond being an "efficient expert consultant telling the learner what to do mainly to ensure excellent patient care" [12]. Giving advice regarding clinical care is not the same as teaching; it misses the crucial step of helping the learners to ask new questions and think critically and creatively about them to "ask new questions, to recognize, and solve new problems" [12] (p. 694).

Furthermore, a substantial investment in faculty development is necessary to modernize our system of medical education. For example, few faculty know the principles of adult learning or how to plan for and moderate teambased discussions in the flipped classroom model, and few have had formal training in moderating small group discussions. Similarly, few faculty have had formal instruction in curriculum design models or have a full appreciation of where their component of education fits into the wider medical curriculum. Most of our senior and midcareer faculty received little training as teachers and were mostly exposed to the standard power point lecture or didactic seminars. Few were trained in eliciting feedback and incorporating it into their academic and clinical teaching.

Assuming that we will continue to need actual face-to-face teaching, the argument is sometimes made that in the case of graduate medical education, the cost of teaching residents still results in a net financial gain given their clinical service. In one review, however, Grover et al. disputed the idea that residents make money for academic medical centers, suggesting that there are no empirical analyses demonstrating that trainee contributions to clinical revenue offset the investment required for training (it is worth noting that the calculations may be different depending on whether the analysis includes subspecialty fellows who are already board certified in their core specialty) [13] (p. 2361).

How this investment is paid for can vary considerably. If the faculty member has time that is underwritten by a salary dedicated to teaching, then the institution (or the department) is paying. If not, the faculty member provides this time over and above their compensated duties, and it is essentially pro bono-in other words, the faculty member pays - usually for the reward of an academic appointment. This constitutes a financial stress which may be particularly difficult for young faculty who have been graduating with an escalating student loan burden. If that stress is more than can be tolerated, the faculty member will likely burnout and the time spent teaching may decrease. One outcome of stretching the system too thin is a decline in quality of the actual educational experience. As a result, the learners pay as well. 


\section{Historical Factors}

For perspective, it may be helpful to review the history of the "academic physician" and how individuals in this role came to be stretched so thin. Ludmerer [8] noted that the early part of the twentieth century saw an exponential increase in the amount of medical knowledge and a corresponding increase in emphasis in clinical reasoning. It was in this context that Flexner advocated in 1930 for a shift to salaried faculty who would make teaching a priority and "learners became the central focus of the faculty's attention" [8] (p. 26). This manifested as a shift from curricula largely comprised of lectures to the addition of clerkships, laboratory instruction, small group conferences, and seminars. Barzansky and Kenagy [14] emphasized the pivotal influence of Flexner, but they focused on his appreciation of the financial pressures on faculty at the time (as opposed to the changing needs of learners). From this view, salaried, full-time faculty "[protected] faculty members from the pressure to generate their own income through clinical practice and so [allowed] them to pursue teaching and research free from distraction" [14].

Flexner thought that salaries would still be less than the potential income of private practice, but that some sacrifice for the mission was a reasonable expectation and that the gratification of contributing to the development of young physicians would compensate for low reimbursement. He argued that his proposed salary structure would not be covered by tuition alone but would require university endowments and philanthropy. The significant introduction of federal funding for research in the 1940s served to underwrite teaching time. Given the difficulties of consistently obtaining funding, however, the model evolved by the1940s-1950s into a mix of fulltime salaried faculty as well as those who supplemented their income through private work.

The creation of Medicare in the 1960s was the next "game changer," since ward patients of faculty became a reliable billing target with private insurance for the first time following Medicare's lead. Barzansky and Kenagy [14] noted that, "clinical practice income came to be an explicit and increasingly important source of medical school revenue" (p. 262), and the portion of revenue from faculty practice increased from 3 to $31 \%$ between 1960 and 1990 . As hospital costs rose, the pendulum eventually swung in the direction of cost containment, and the rise of managed care led to a new emphasis on clinical productivity for faculty, with financial incentive for seeing more patients. It should also be noted that the legacy of the managed care movement led to a $20 \%$ reduction in graduate medical education (GME) slots nationally, shorter hospital stays, and larger patient panels for physicians of all specialties.

The decrease in GME slots, of course, resulted in a diminished workforce both for patient care and teaching. Unfortunately, Ludmerer noted that, "educational activities have simply not been encouraged by the new rules of faculty practice that penalize any loss of "clinical productivity"' [8] (p. 27).To counter these financial pressures, schools have provided some explicit compensation for teaching, generally to departments, and have created teaching academies to augment prestige. Yet, Barzansky and Kenagy noted that, "The current salaried full-time faculty member is highly dependent on clinical practice for his or her income" and "vulnerable to time pressures that mitigate their ability to participate in teaching" [14] (pp. 264, 263).Given the lowered reimbursements and increased patient demands, many devoted medical teachers were caught in avise of increased clinical demands and unfunded pressure to maintain their teaching excellence.

Assessments of the impact of these clinical productivity demands on teaching have yielded mixed results. One review examined published reports on the impact of productivity incentives in academic medical centers and found only three that examined the effect of the program on teaching evaluations or educational quality, with each reporting no change in these outcomes [15]. The authors noted, however, that "two of these programs provided a credit for time spent teaching and the third set an expectation for faculty participation in educational activities," thus explicitly attempting to mitigate the impact of the productivity demands (p. 257). Another group reviewed eight studies that linked compensation to productivity measures (clinical, research, and teaching) and found that the plans "appeared to improve productivity in research activities, possibly improved clinical productivity but had no effect in the area of teaching" [16] (p. 5). By contrast, a more recent study in a large urban academic internal medicine department that had recently implemented clinical productivity work targets found that $86 \%$ of faculty reported being less likely to engage in activities that did not directly count toward productivity targets, and $75 \%$ specifically reported a decrease in time spent teaching. The authors noted that $35 \%$ of optional written comments in the survey also mentioned some concern over impact on education [17].

In short, decades ago, faculty were more likely to welcome opportunities for teaching as their reimbursements, patient panels, and productivity demands allowed more margin for the mission of education. An additional stress on faculty is decreased time with other physicians. It may sound nostalgic, but many of us recall the time we used to spend together. We enjoyed participating in a professional community whether at conferences, on rounds, and in clinics discussing cases. Much of this time was invaluable for interpersonal connection, developing a shared mission, and for "curbside" education. These benefits reinforced morale and diminished burnout. These times are now either minimally existent or considered luxuries. The rapid pace and demands of modern medicine have fragmented the reality of a community of healthcare professionals and trainees, no doubt contributing to today's high burnout rate. 


\section{Some Possible Solutions}

Partly driven by a concern over the prioritization of clinical productivity, some academic medical centers began adopting "mission-based management" programs that defined and tracked education efforts as championed by the Association of American Medical Colleges (AAMC). A review in 2002 identified 41 schools that were using some kind of metric to quantify teaching activity [18]. Structured interviews with the schools' leadership identified a number of different and sometimes conflicting objectives for these projects: (1) to develop a systematic way to distribute funding, (2) to track the resources spent on teaching for the purpose of required reporting, (3) to address chairs' mistrust of the dean's office, (4) to counter the "myth" that faculty cannot afford to teach or are not compensated, (5) to provide incentives to teach, and (6) to raise the visibility and thus the value of teaching. The authors found that most schools did not have a culture of using data for education or salary decisions and that faculty expressed anxiety that the data would be used in a clumsy or harmful fashion. The authors also noted that efforts often stalled because the school was trying to find the perfect metric that could account for all aspects of teaching and included quality measures.

We can approach the task of creating a "good enough" plan to account for and support teaching efforts by outlining a set of critical decisions for the institution to make. First, will there be a relative value assigned to different teaching activities reflecting the institutional priorities? For example, the Medical Education Panel of the Mission-based Management Program of the AAMC devised a relative value scale since they felt that equating faculty performance with time spent on a teaching activity was inadequate [19]. The result was a complicated four-step process to create Relative Value Units (RVUs) for education. This entailed creating a comprehensive list of education activities and assigning a weight to each, modifying this by quality of performance, adjusting by variable weighting for different categories of educational work (teaching, scholarship in education, development of education products), and then factoring in the relative importance of the educational program to the school's mission.

Given how little traction this proposal attained, it is probably critical to keep the system simple. One medical school reported mission-based planning that did not attempt to assign relative value (or clinical productivity) but instead focused on the raw cost of each mission in terms of time [20]. The school used a survey of the faculty to define the amount of time spent in each activity, including teaching. When faculty were engaged in an activity that was a mix of clinical work and teaching or research and teaching, the planning group decided to designate $50 \%$ of this time to teaching, which may have been an overly generous allocation. They also decided to designate a set amount of hours to lecture preparation (as opposed to leaving this up to faculty discretion and self-report). The authors reported an improved understanding of the actual costs of the missions and better linkage of faculty time (i.e., cost) to each mission.

A second decision involves what exactly to include under teaching time. Many programs include preparation time for teaching, such as in the preceding example, while others do not. Most include both classroom and bedside teaching, but some programs feel that bedside teaching is already accounted for. For example, in an academic RVU system created for a radiology department, the RVU for teaching included lectures but did not cover teaching in the course of clinical work, as this is "already included in the clinical work RVU" [21]. However, the RVU for teaching in this plan did include a modifying factor based on learners' evaluation score. The question of whether to include a quality measure is a third decision point and seems to be relatively uncommon.

Finally, a fourth decision involves the process of collecting and collating the data used to estimate teaching effort. The accounting of teaching time can be derived from self-reporting of faculty (i.e., what are they currently doing) or from an institution's best estimate of the teaching needs. Although most reports begin by surveying faculty about their time expenditures, Jarrell et al. argued against starting with the individual faculty member's self-report of teaching activities [22]. Instead, they described a process for calculating how much time would be required for medical student education at a maximum in delivering didactics and face-toface teaching in clinical care delivery settings. Their concern was to avoid the potential biases and self-interest in selfreports and to ensure a focus on the institution's education priorities.

In measuring and tracking how faculty time is spent, a department may find a poor correlation between salary support for education and actual time spent in education. Stites et al. [23] considered creating an RVU system with value assigned for predefined educational tasks, but found it rapidly became too arbitrary and complex. Instead, they decided to institute self-report logs of educational time (lecturing or preparing to teach), with some pre-assigned time allocations set for specific educational administrative roles like course or clerkship director and associate program director. They added a factor for time spent as a clinical attending on services where clinical productivity would be presumably decreased by supervision/teaching duties. They then added up all this time and divided the university's total funding for education for the department according to the individual faculty's time reported in education. There was no net change in how much money was going to education or in total salary of each faculty member, but there was a huge shift in who was getting money for education, and therefore how much was left to be covered by clinical productivity. A survey of the faculty's perception of this system showed significant improvement in their view that 
university support was now better aligned with actual educational effort.

There has been little published specifically about the experience of academic psychiatry departments in evaluating the time and effort for teaching. A group at the University of California, Davis described a method for reporting, reviewing, and setting expectations for percentages of faculty's time allocated to each educational mission. In their plan, credit for teaching activities was given as percentages of base salary. For example, one contact hour per week of individual resident supervision was worth $2 \%$ of base salary, while directing the second year medical students' psychiatry course was worth $20 \%$. Credit for less regular teaching, such as seminars and lectures, was given based on the number of actual contact hours. Unfortunately, the impact on educational productivity or faculty satisfaction was unclear [24, 25].

Clearly, there is no perfect method to calculate, monitor, and protect the time needed to teach. Perfect should not be the enemy of good - any approach made by the institution and the psychiatry department is better than none at all. Each academic medical center should be expected to develop some plan to ensure that teaching, including clinical supervision, is protected and compensated. This can be achieved in several ways: (1) there can be allocation of salary support for a percentage the faculty's time, (2) specific teaching tasks can be designated for specific compensation, or (3) the faculty member's clinical productivity requirements can be decreased to create some margin in their schedule for teaching. The clinical productivity expectations of an attending who is also supervising medical students and residents in the clinic and ward should be reduced by some significant percentage when compared with a non-teaching hospitalist.

Further, a plan must be developed to account for ongoing faculty assessment and quality improvement. Requirements that faculty track and report their educational time should be supported. One can easily object that creating another reporting requirement will itself lead to increased bureaucracy and more burnout. Reporting requirements should therefore be coupled with actual protection of teaching time so that there is a net improvement in time spent doing meaningful and fulfilling work. In addition, use of integrated suites of educational software that cover both undergraduate and graduate medical education at some institutions should be used to autopopulate these reports whenever possible.

An important aspect of an institution's culture is created by what is monitored and tracked, generating a "hidden curriculum" of faculty development that lets the faculty know what is truly valued in their department. This system should include measures of teaching quality that include learner assessment, peer review, and learner outcomes. Beyond monitoring, we need to foster a new culture of practice based on learning and improvement in our faculty. With time built into salary structures, a thoughtful educational program for faculty at all levels should be an integral part of each department's structure. A Grand Rounds series, common to most departments, is insufficient for a change in culture. We need a new curriculum for ongoing education and development of faculty. This should not simply satisfy the current Maintenance of Certification requirements, but rather should be meaningful for the ongoing education of our faculty as teachers. Thus, faculty need time both to teach and to learn how to teach.

If the task is to make sure that there is adequate time to teach and learn, perhaps the most critical issue is how we conceptualize ownership of this task. Unless we want teaching to simply add pressure and paradoxically foster burnout, academic medical centers must take ownership of the issue by instituting systems of tracking, compensating, and protecting teaching time. In other fields where lives and safety are at high stake (e.g., aviation or certain military operations), there would be little question of either the time or other resources needed to assure minimum standards of competence. Yet chairs, program directors, and other educational leaders almost have to apologize for needing to request enough positions to account for protected teaching and program administration time. It is crucial that the responsibility be placed at the institution and not at the departmental level, because there is often great disparity among departmental finances. Some specialties, particularly those that have significant procedural interventions, constitute the "haves" while departments that encompass primary care or prioritize face-to-face time with patients constitute the "have not's." Shifting the ownership of the problem to the institution would be a significant step in decreasing the stress on young faculty. This is likely to have far more impact in preventing burnout than faculty wellness programs, particularly, if it results in the experience of a community of faculty working and learning together.

We do not need to be pessimistic about the possibility of meaningful institutional change. A study at Harvard's primary care clerkship found that increasing the preceptors' stipend so that it actually compensated for lost time was associated with markedly improved retention. The authors noted that their survey of the preceptors showed that the stipend was not the strongest motivator or source of satisfaction (having a good student was); instead, the pay "eases the burden of teaching in a busy practice such that the intrinsic pleasure of teaching is given play" [26] (p. 46).In other words, decreasing the financial stress allowed space for the revitalizing experience of meaningful work as an educator.

One of the ironies of many faculty development initiatives is that while they are likely to encompass sessions on teaching skills, they simultaneously may provide burnout prevention in the form of "time management" skills, that include empowering the junior faculty to say "no." Unfortunately, that "no" may be directed at requests to teach another seminar, or supervise another psychotherapy trainee, or prepare another lecture. If we want to ensure both excellent education and simultaneously 
prevent burnout, we hope that faculty will be able to say "yes" to activities that provide greater meaning and satisfaction to their work with the confidence that they will be administratively and financially supported in this mission.

\section{Compliance with Ethical Standards}

Disclosure On behalf of all authors, the corresponding author states that there is no conflict of interest.

\section{References}

1. Accreditation Council for Graduate Medical Education. ACGME common program requirements section VI with background and intent. Access. Oct 2017:24. http://acgme.org/Portals/0/PFAssets/ ProgramRequirements/CPRs_Section\%20VI_with-Backgroundand-Intent_2017-01.pdf

2. Krasner MS, Epstein RM, Beckman H, et al. Association of an educational program in mindful communication with burnout, empathy, and attitudes among primary care physicians. JAMA. 2009;302:1284-93.

3. Wallace JE, Lemaire JB, Ghali WA. Physician wellness: a missing quality indicator. Lancet. 2009;374:1714-21.

4. Shanafelt TD, Drybye LN, West CP. Addressing physician burnout: the way forward. JAMA. 2017;317:901-2.

5. Shanafelt TD. Enhancing meaning in work: a prescription for preventing physician burnout and promoting patient-centered care. JAMA. 2009;302:1338-40.

6. Pololi LH, Evans AT, Civian JT, et al. Faculty vitality—surviving the challenges facing academic health centers: a national survey of medical faculty. Acad Med. 2015:930-6.

7. Accreditation Council for Graduate Medical Education. Clinical Learning Environment Review (CLER). Access. Oct 2017:24. http://acgme.org/WhatWeDo/Initiatives/ClinicalLearning EnvironmentReview (CLER)?articleid=3841

8. Ludmerer KM. Time and medical education. Ann Intern Med. 2000;132:25-8.

9. Barzansky B. United States medical school financing: beyond the black box. Medical Science Educator. 12
10. Dzau VJ, Cho A, ElLaissi W, et al. Transforming academic health centers for an uncertain future. NEJM. 2013;369:991-3.

11. Kahn MJ, Maurer R, Wartman SA, Sachs BP. A case for change: disruption in academic medicine. Acad Med. 2014;89:1216-9.

12. Skeff KM, Bowen JL, Irby DM. Protecting time for teaching in the ambulatory care setting. Acad Med. 1997;72:694-7.

13. Grover A, Slavin PL, Willson P. The economics of academic medical centers. NEJM. 2014;370:2360-2.

14. Barzansky B, Kenagy G. The full-time clinical faculty: what goes around, comes around. Acad Medicine. 2010;85:260-5.

15. Andreae MC, Blad K, Cabana M. Physician compensation programs in academic medical centers. Health Care Mange Rev. 2006;31:251-8.

16. Akl EA, Meerpohl JJ, Raad D, et al. Effects of assessing the productivity of faculty in academic medical centres: a systematic review. Can Med Assoc J. 2012; https://doi.org/10.1503/cmaj.111123.

17. Summer R, Wiener RS, Carroll D, Sager A. Physician perception of the impact of productivity measures on academic practice. Arch Intern Med. 2012;172:967-9.

18. Mallon WT, Jones RF. How do medical schools use measurement systems to track faculty activity and productivity in teaching? Acad Med. 2002;77:115-23.

19. Nutter DO, Bond JS, Coller BS, et al. Measuring faculty effort and contribution in medical education. Acad Med. 2000;75:199-207.

20. Sloan TB, Kaye CI, Allen WR, Magness BE, Wartman BA. Implementing a simpler approach to mission based planning in a medical school. Acad Med. 2005;80:994-1004.

21. Mezrich R, Nagy PG. The academic RVU: a system for measuring academic productivity. J Am CollRadiol. 2007;4:471-8.

22. Jarrell BE, Mallot DB, Peartree LA, Calia FM. Looking at the forest instead of counting the trees: an alternative method for measuring faculty's clinical education effort. Acad Med. 2002;77:18-23.

23. Stites S, Vansaghi L, Pingleton S, Cox G, Paolo A. Aligning compensation with education: design and implementation of the educational value unit (EVU) system in an academic internal medicine department. Acad Med. 2005;80:1100-6.

24. Anders TF, Hales RE, Shakrokh NC, Howell LP. Mission based reporting in academic psychiatry. Acad Psych. 2004;28:129-35.

25. Hales RE, Shahrokh NC, Servis M. A department of psychiatry faculty practice plan designed to reward educational and research productivity. Acad Psych. 2005;29:244-8.

26. Peters AS, et al. How important is money as a reward for teaching? Acad Med. 2009;84:42-6. 